\title{
Universiteit
}

Leiden

The Netherlands

\section{Spatial labeling in a two-photon interferometer}

Lee, P.S.K.; Exter, M.P. van

\section{Citation}

Lee, P. S. K., \& Exter, M. P. van. (2006). Spatial labeling in a two-photon interferometer.

Physical Review A, 73, 063827. doi:10.1103/PhysRevA.73.063827

Version: $\quad$ Not Applicable (or Unknown)

License: $\quad$ Leiden University Non-exclusive license

Downloaded from: https://hdl.handle.net/1887/61322

Note: To cite this publication please use the final published version (if applicable). 


\title{
Spatial labeling in a two-photon interferometer
}

\author{
P. S. K. Lee and M. P. van Exter \\ Huygens Laboratory, Leiden University, P.O. Box 9504, 2300 RA Leiden, The Netherlands
}

(Received 31 March 2006; published 28 June 2006; corrected 6 July 2006)

\begin{abstract}
We study the spatial coherence of entangled photon pairs that are generated via type-I spontaneous parametric down-conversion (SPDC). By manipulating the spatial overlap between the two down-converted beams in a Hong-Ou-Mandel interferometer we observe the spatial interference of multiple transverse modes for an even and an odd number of mirrors in the interferometer. We demonstrate that the two-photon spatial coherence, which is quantified in terms of a transverse coherence length, differs completely for the two mirror geometries and support this result by a theoretical and experimental explanation in terms of photon labeling.
\end{abstract}

DOI: 10.1103/PhysRevA.73.063827 PACS number(s): 42.50.Ar, 42.65.Lm, 03.67.Mn, 42.50.Dv

\section{INTRODUCTION}

In the last two decades, the use of entangled photon pairs has become a popular tool for several experimental studies on both the foundations [1-3] and applications [4,5] of quantum mechanics. One of the most fascinating among these experiments has been introduced by Hong, Ou, and Mandel in order to measure the coherence length of a two-photon wave packet produced under spontaneous parametric down conversion [2]. In this original two-photon interference experiment, which we will simply call the HOM experiment, two entangled photons that arrive simultaneously at the two input ports of a beamsplitter will effectively bunch and together exit one of the two output ports. As a consequence, no coincidence events are measured between photon detectors placed in each output channel. As soon as the two photons become distinguishable due to a time delay between the two input beams, the coincidence rate will reappear. Therefore, the coincidence rate measured as a function of the relative time delay shows a minimum at zero delay, which is now known as the HOM dip.

Pittman et al. [6] showed that HOM interference is also possible if the two photons arrive at different times at the beamsplitter, provided that the detectors cannot distinguish one probability path from another; the interference actually occurs between the two probability paths of the photon pair and not between the individual photons. Rarity and Tapster [7] demonstrated that two-photon (HOM) interference is even possible between two uncorrelated photons from independent sources. This experiment, which has been repeated by several groups $[8,9]$, is however only possible if the two photons are completely indistinguishable. More precisely, these photons must arrive at the same time (within the inverse detection bandwidth) and in the same spatial mode. Experimentally, this requires pulsed pumping [7] and singlemode (fiber-coupled) detection, respectively. In case of $\mathrm{cw}$ pumping, the existence of two-photon interference is in fact a proof of time entanglement; while the individual arrival times of the photons in the generated pairs are undetermined, these two times are strongly quantum correlated. If the detectors observe many transverse modes, a similar argument shows that two-photon interference is only possible if the two photons are spatially entangled; while the spatial profiles of each of the photons is undetermined, a measurement on one photon codetermines the position and momentum of the other.

Since its initial demonstration in 1987, the HOM interferometer has been employed in several experimental schemes. Like the original experiment, most of these HOM experiments focus merely on the temporal coherence of the twophoton wave packet [9-11]. Only recently, some papers have reported on the spatial aspects of the HOM experiment [12-14]. Walborn et al. [12] have demonstrated how the transverse spatial symmetry of the pump beam affects the two-photon interference: for a symmetric two-photon polarization state, one can make the transition from a HOM dip to HOM peak by changing the pump profile from even to odd. Caetano et al. [13] and Nogueira et al. [14] have performed coincidence imaging experiments, measuring the coincidence rate behind two small detectors as a function of their transverse position. Using an antisymmetric pump profile, they observed spatial antibunching of the two photons in the coincidence image.

So far, all reported experiments have used perfect spatial overlap between the signal and idler beams and studied the two-photon interference mostly as a function of the temporal delay in the HOM interferometer. Spatial aspects of a HOM interferometer, in a collapsed type-II collinear geometry, have been studied via the shape, size, and displacement of the detection apertures, but the generated beams remained unchanged [15]. The effect of a possible size difference between two nonoverlapping beams has been studied theoretically in few-photon interference [16], but beam displacements were not considered. In this paper, we will present the first experimental results on two-photon interference under the influence of a physical separation of the signal and idler beams in the transverse plane. For this purpose, we have used a more general HOM interferometer which employs not only a longitudinal but also a transverse displacement of one beam with respect to the other.

By measuring coincidences as a function of the beam displacement we determine the transverse coherence length of the two-photon wave packet for different detection geometries, i.e., different numbers of interfering transverse modes. The key question is how the two-photon spatial coherence manifests in an interferometer with either an even or an odd number of mirrors in the combined signal and idler path. We find that the mirror geometry of the interferometer does indeed play a crucial role. When the total number of mirrors is 
even, the observed spatial interference is sensitive only to the sum of both coordinates and thereby to the profile of the pump. In case of an odd number of mirrors, one probes the two-photon coherence in the difference coordinate, and thereby basically observes the spherical wavefronts of point sources. Most of our experiments have been performed with an odd number of mirrors, a geometry that has not been studied before.

This paper is organized as follows. In Sec. II we present a theoretical description of two-photon (HOM) interference for both an even and an odd mirror geometry, including both temporal and spatial degrees of freedom. Our experimental results can be found in Sec. III, which is split into the following sections. After introducing the experimental setup in Sec. III A, we present our experimental results on temporal labeling in Sec. III B and on spatial labeling in Sec. III C. In Sec. III D we analyze the spatial aspects from a different perspective, using a discrete modal basis. We end with a concluding discussion in Sec. IV.

\section{THEORETICAL DESCRIPTION}

\section{A. The generated two-photon field}

The calculation of the two-photon interference observed in a general HOM interferometer, with a combined temporal delay and transverse spatial shift in one of the arms, is mainly a matter of good bookkeeping. This bookkeeping deals to a large extent with the coordinate changes between two reference frames. The laboratory frame, having its $z$ axis along the pump beam and the surface normal of the crystal, is the natural choice for the generated field. The two local beam frames that are oriented along the two beam directions are the natural coordinate systems at the detectors. To simplify the notation we will display only one spatial direction, being the $x$ coordinates in the plane through the signal and idler beam.

We consider two-photon emission by spontaneous parametric down-conversion (SPDC) in the so-called thin-crystal limit, where the detected space angle and spectral bandwidth must be much smaller than the generated SPDC ring size and bandwidth, respectively. In this limit, the generated twophoton wave function is [17]

$$
\psi_{z}\left(x_{s}, x_{i} ; \Delta \omega\right)=\int E_{p}(x) h\left(x_{s}, x ; \omega_{s}\right) h\left(x_{i}, x ; \omega_{i}\right) d x,
$$

where $E_{p}(x)$ is the field profile of the pump beam at $z=0$, and $x_{s}$ and $x_{i}$ are transverse coordinates in the laboratory frame. The one-photon propagators $h\left(x_{s}, x ; \omega_{s}\right)$ $=1 /\left(i \lambda L_{s}\right)^{2} \exp \left(i k_{s} L_{s}\right)$ and $h\left(x_{i}, x ; \omega_{i}\right)=1 /\left(i \lambda L_{i}\right)^{2} \exp \left(i k_{i} L_{i}\right)$ describe the propagation of the signal and idler photon from the crystal to the detection plane. They contain the wavevector amplitudes $k_{s, i}=\omega_{s, i} / c$ and the path lengths $L_{s, i}$. We will consider almost frequency-degenerate SPDC, where the frequency difference $\Delta \omega \equiv \omega_{s}-\omega_{i}$ and where the sum $\omega_{s}$ $+\omega_{i}=\omega_{p}=c k_{p}$ is fixed by the quasimonochromatic pump.

Next we introduce "beam coordinates" $\delta x_{s}$ and $\delta x_{i}$ that

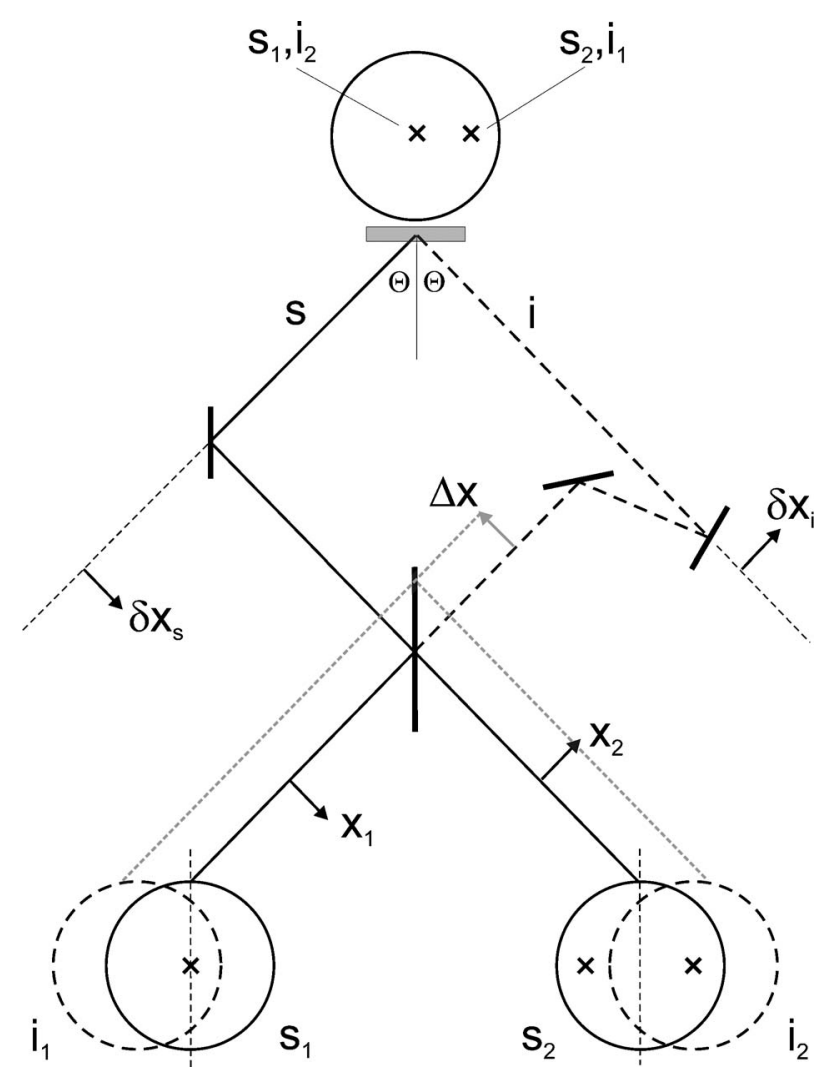

FIG. 1. Optical-path geometry of a HOM interferometer with one mirror in the signal beam and two mirrors in the idler beam, which also contains a displacement $\Delta x$. The five circles denote the pump spot and four possible images thereof. These are used to explain the occurrence of spatial labeling (see Sec. III C for details).

are defined with respect to the two beam axes in the signal and idler direction, which themselves are oriented at angles $-\Theta$ and $\Theta$ with respect to the pump laser (see Fig. 1). Beam coordinates are more convenient to evaluate the effect of beam reflections and translations and have the extra advantage that the coordinates $\delta x_{s, i}$ remain relatively small. Substitution of $\delta x_{s, i}$ for $x_{s, i}$ in Eq. (1) immediately yields the generated two-photon wave function in beam coordinates. Working in the paraxial limit, we expand the path lengths as $L_{s, i} \approx L+\left|\delta x_{s, i}-x\right|^{2} / 2 L \pm x \Theta$. The term $\pm x \Theta$ describes how a displacement at the crystal leads to a change of the signal and/or idler path on account of the viewing angle.

By comparing the combined propagator of the two-photon field with the one-photon propagator of the pump field to a detection plane at a distance $L$ behind the crystal, we can solve the integration in Eq. (1) to obtain the relatively complicated expression

$$
\begin{aligned}
\psi\left(\delta x_{s}, \delta x_{i} ; \Delta \omega\right) \approx & E_{p, z}\left(\frac{1}{2}\left(\delta x_{s}+\delta x_{i}\right)-\gamma\right) \\
& \times \exp \left(\frac { i k _ { p } } { 8 L } \left[\left|\delta x_{s}-\delta x_{i}\right|^{2}\right.\right. \\
& \left.\left.+4 \gamma\left(\delta x_{s}+\delta x_{i}\right)-4 \gamma^{2}\right]\right),
\end{aligned}
$$


where $E_{p, z}$ is the pump profile in the detection plane [18] and $\gamma=L \Theta \Delta \omega / \omega_{p}$ is a transverse displacement that appears only for $\Delta \omega \neq 0$. The approximation is almost perfect and only refers to the removal of a small phase term $(\ll 1)$ of the order of $\left(\Delta \omega / \omega_{p}\right)^{2}$ times the Fresnel number $N_{F}$ of the detected system.

Equation (2) gives a full description of the spatial and temporal coherence of the generated two-photon field in the considered thin-crystal limit. It shows among others that this field has a completely different spatial coherence in the sum coordinate $\delta x_{s}+\delta x_{i}$ than in the difference coordinate $\delta x_{s}$ $-\delta x_{i}$. Whereas the former is dictated by the profile of the pump laser, the latter is characterized by the field curvature of a point source. This difference is of vital importance in the rest of our discussion and causes the very different behavior of two-photon interferometers with an even or odd number of reflecting mirrors (see Sec. II C).

If the detection bandwidth is too large to satisfy the quasimonochromatic limit, we should include the effects of $\gamma$ $\neq 0$ in our discussion of Eq. (2). These effects are discussed in Sec. II D. For the moment we will simply explain their origin. The extra phase terms originate from the comparison of the $[\exp (i k L)$ terms in the] propagators of signal, idler and pump beams. The argument of the pump profile $E_{p, z}$ depends on $\Delta \omega$, because this argument can also be written as the weighted sum $\left(k_{s} x_{s}+k_{i} x_{i}\right) / k_{p}$ of the signal and idler positions $x_{s}$ and $x_{i}$ in the laboratory frame [18]. In the nonmonochromatic limit, the spatial and spectral degrees of freedom become mixed, basically because the transverse momenta of the signal and idler photon depend both on their emission angle $(\approx \mp \Theta)$ and photon frequency $\omega$.

\section{B. Two-photon interference}

In a standard (HOM) two photon interferometer the signal and idler beam are combined on a beamsplitter of which the two output beams are filtered spectrally and spatially, before being detected by two-photon detectors. The observed twophoton interference is most easily described in the beam coordinates $x_{1}$ and $x_{2}$ of the two local coordinate systems that are centered around the two axes at detectors 1 and 2, respectively. We thus need to express the detected two-photon field $\psi_{\mathrm{det}}\left(x_{1}, x_{2} ; \Delta \omega_{12}\right)$ (with $\left.\Delta \omega_{12}=\omega_{1}-\omega_{2}\right)$ in terms of the generated field. As coincidence counts in a HOM interferometer can be generated by two possible routes, being either a reflection of both signal and idler photon at the beamsplitter or a double transmission, we can symbolically express the detected two-photon field as

$$
\psi_{\mathrm{det}}\left(x_{1}, x_{2} ; \Delta \omega_{12}\right)=-R \psi_{r r}(\cdots)+T \psi_{t t}(\cdots),
$$

where the intensity reflection $R$ and transmission $T$ are equal to $\frac{1}{2}$ only for the ideal beamsplitter. The coordinates in the two-photon fields $\psi_{r r}$ and $\psi_{t t}$ are left out on purpose. One reason for this is that the transformation from detector to crystal coordinates is different for the two possible routes.

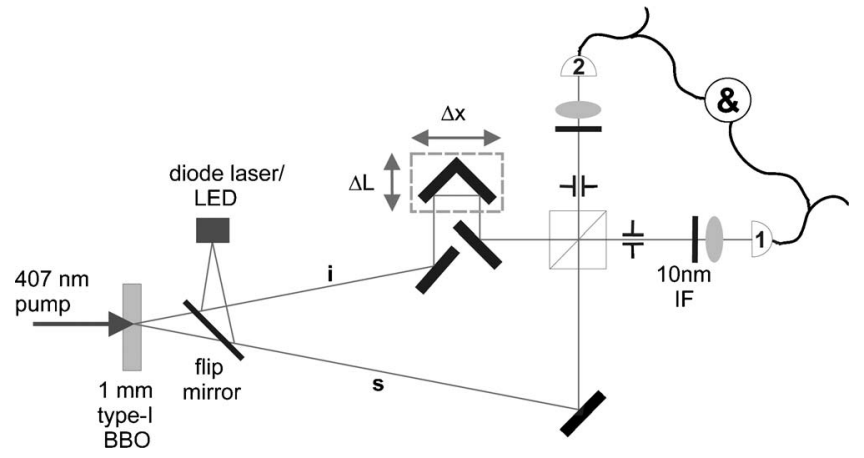

FIG. 2. Schematic view of the experimental setup (see text for details).

Another reason is that the actual transformation also depends on the number of mirrors and on the time delay $\Delta t=\Delta L / c$ and transverse displacement $\Delta x$ imposed in one of the interferometer arms.

The coincidence count rate $R_{c}$ observed behind spatial apertures and spectral filters is found by integrating $\left|\psi_{\text {det }}(\cdots)\right|^{2}$ over the corresponding spatial and spectral coordinates, as

$$
R_{c}=\int d \omega_{1} d \omega_{2} d x_{1} d x_{2}\left|\psi_{\mathrm{det}}\left(x_{1}, x_{2} ; \Delta \omega_{12}\right)\right|^{2}
$$

The interference between the two-photon fields $\psi_{r r}$ and $\psi_{t t}$ is contained in the cross terms of $\left|\psi_{\text {det }}\right|^{2}$. This interference is only present close to zero delay and perfect spatial overlap, but disappears when either $\Delta t$ or $\Delta x$ are sufficiently large. In general we can thus write the coincidence count as

$$
R_{c}(\Delta t, \Delta x)=R_{c, \infty}\left(1-\frac{2 R T}{R^{2}+T^{2}} V_{\mathrm{HOM}}(\Delta t, \Delta x)\right) .
$$

In the rest of the discussion we will concentrate on the temporal and spatial dependence of the visibility function $V_{\mathrm{HOM}}(\Delta t, \Delta x)$, which contains the interesting physics. The factor $V_{R T}=2 R T /\left(R^{2}+T^{2}\right)$ just specifies the "intensity unbalance" between the two probability channels. The visibility function

$$
V_{\mathrm{HOM}} \approx \frac{\operatorname{Re}\left[2\left\langle\psi_{r r} \mid \psi_{t t}\right\rangle\right]}{\left\langle\psi_{r r} \mid \psi_{r r}\right\rangle+\left\langle\psi_{t t} \mid \psi_{t t}\right\rangle},
$$

basically measured the spectral overlap between the twophoton fields $\psi_{r r}$ and $\psi_{t t}$, where we have used the shorthand notation $\langle\cdots\rangle=\int d \omega_{1} d \omega_{2} d x_{1} d x_{2}$. Alternatively, one could say that $V_{\text {HOM }}$ measured the overlap between one two-photon field $\left(\psi_{r r}\right)$ and a modified version thereof $\left(\psi_{t t}\right)$, and can thereby provide information on the spatial and/or temporal coherence of this field. The physical interpretation of the visibility function $V_{\mathrm{HOM}}$ is that it quantifies the amount of temporal and/or spatial labeling of the two photons. If any 
properties of the detected photons 1 and 2 allow one to decide which photon took the signal path and which photon took the idler path, this so-called labeling will remove the interference between the two probability channels.

\section{Why the number of mirrors matters}

In this section we will highlight the difference between two-photon interferometers with an even or odd number of reflecting mirrors in the combined signal and idler path by presenting detailed expressions of $V(\Delta t, \Delta x)$ for both cases. Based on these general expressions, Secs. II D and II E will separately treat the occurrence of temporal labeling $\left[V_{\mathrm{HOM}}(\Delta t)\right.$ at $\left.\Delta x=0\right]$ and spatial labeling $\left[V_{\mathrm{HOM}}(\Delta x)\right.$ at $\Delta t$ $=0]$, again using the distinction between an even and odd number of reflections.

Figure 1 depicts a possible HOM interferometer, which in this case has one mirror in the signal path and two mirrors in the idler path and thus falls in the "odd" category. It is also a sketch of the experiment, where we use $1+4$ mirrors. The idler path contains an adjustable transverse displacement $\Delta x$ (as shown) and an additional longitudinal displacement $\Delta L=c \Delta t$ (shown only in the experimental setup of Fig. 2). The beams are labeled such that the doubly reflected path links the coordinate indices $(s \leftrightarrow 1)$ and $(i \leftrightarrow 2)$, making $\Delta \omega$
$=\Delta \omega_{12}$, whereas the doubly transmitted path links $(s \leftrightarrow 2)$ and $(i \leftrightarrow 1)$, making $\Delta \omega=-\Delta \omega_{12}$. The crucial point to note, and the whole reason for the "odd or even" distinction, is that every additional reflection in either signal or idler path leads to an inversion of the corresponding beam coordinate $\delta x \leftrightarrow-\delta x$.

We will first consider an interferometer with one mirror in the signal and one mirror in the idler path, i.e., with an even number of mirrors. For this balanced interferometer the relation between the detected and generated two-photon field [Eq. (3)] is

$$
\begin{aligned}
\psi_{\text {even }}\left(x_{1}, x_{2} ; \Delta \omega_{12}\right)= & -R \psi\left(x_{1}, x_{2}+\Delta x ; \Delta \omega_{12}\right) e^{i \omega_{2} \Delta t} \\
& +T \psi\left(-x_{2},-x_{1}+\Delta x ;-\Delta \omega_{12}\right) e^{i \omega_{1} \Delta t},
\end{aligned}
$$

where the longitudinal delay $\Delta t$ and transverse displacement $\Delta x$ are both imposed on the idler beam. Note that the arguments in the two contributions $\psi_{r r}$ and $\psi_{t t}$ are related through a swap of the labels $1 \leftrightarrow 2$ in combination with an inversion $x_{j} \leftrightarrow-x_{j}$ (for $j=1,2$ ). Substitution into Eq. (2) shows that the two contributions have the dominant part of the exponential factor in common, as $\delta x_{s}-\delta x_{i}=x_{1}-x_{2}-\Delta x$ for both terms, but differ in the argument in the pump field. For this "even" geometry, the visibility function $V_{\mathrm{HOM}}$ thus becomes

$$
V_{\text {even }}(\Delta t, \Delta x) \approx \frac{\operatorname{Re}\left[2 \int e^{i \Delta \omega_{12} \Delta t} e^{\left(i k_{p} / L\right) \gamma_{12} \Delta x} E_{p, z}^{*}\left(-\alpha+\frac{1}{2} \Delta x\right) E_{p, z}\left(\alpha+\frac{1}{2} \Delta x\right)\right]}{\int\left|E_{p, z}\left(-\alpha+\frac{1}{2} \Delta x\right)\right|^{2}+\left|E_{p, z}\left(\alpha+\frac{1}{2} \Delta x\right)\right|^{2}},
$$

where the integration runs over $x_{1}, x_{2}, \omega_{1}$, and $\omega_{2}$ and where we have introduced $\alpha=-\frac{1}{2}\left(x_{1}+x_{2}\right)+\gamma_{12}$ as help variable, with $\gamma_{12}=L \Theta \Delta \omega_{12} / \omega_{p}$. The sensitivity of $V_{\text {even }}$ to a transverse displacement $\Delta x$ is thus found to be determined mainly by the shape of the pump beam, in combination with the limitations set by the finite integration range over the detection apertures. Especially the symmetry of the pump beam under reflection in the $y z$ plane plays a crucial role. If this beam is symmetric under reflection, the two-photon interference will result in the familiar HOM dip $\left(V_{\mathrm{HOM}}>0\right)$, if this beam is antisymmetric a $\mathrm{HOM}$ peak $\left(V_{\mathrm{HOM}}<0\right)$ will result instead [12].

The above result applies to any geometry where the total number of mirrors in the signal and idler beam is even. Officially, one should still distinguish two subclasses, but these give basically the same result. If both signal and idler beam contain an odd number of mirrors we obtain expressions identical to the ones found above for the case of " $1+1$ mirror." If both signal and idler beam contain an even number of mirrors all positions $x_{j}$ should be inverted, but $V_{\text {even }}$ is again described by Eq. (8) with a new $\alpha=-\frac{1}{2}\left(x_{1}+x_{2}\right)-\gamma_{12}$.

Next we consider the interferometer of Fig. 1, which contains one mirror in the signal path and two mirrors in the idler path, and thus falls in the "odd" category. For this unbalanced interferometer, the relation between the detected and generated two-photon field [Eq. (3)] is

$$
\begin{aligned}
\psi_{\text {odd }}\left(x_{1}, x_{2} ; \Delta \omega_{12}\right)= & -R \psi\left(x_{1},-x_{2}-\Delta x ; \Delta \omega_{12}\right) e^{i \omega_{2} \Delta t} \\
& +T \psi\left(-x_{2}, x_{1}-\Delta x ;-\Delta \omega_{12}\right) e^{i \omega_{1} \Delta t},
\end{aligned}
$$

which differs from Eq. (7) only by a sign in the idler coordinate $\delta x_{i}$. Substitution into Eq. (2) shows that the two terms now have slightly different exponential factors, but almost identical arguments in the pump field, as the combination $\delta x_{s}+\delta x_{i}$ is the same for both $\psi_{r r}$ and $\psi_{t t}$. For this "odd" geometry, the visibility function $V_{\mathrm{HOM}}$ is 


$$
V_{\text {odd }}(\Delta t, \Delta x) \approx \frac{\operatorname{Re}\left(2 \int e^{i \Delta \omega_{12} \Delta t} e^{-\left(i 2 k_{p} / L\right) \gamma_{12} \beta} e^{-\left(i k_{p} / 2 L\right)\left(x_{1}+x_{2}\right) \Delta x} E_{p, z}^{*}\left(\beta-\gamma_{12}\right) E_{p, z}\left(\beta+\gamma_{12}\right)\right)}{\int\left|E_{p, z}\left(\beta-\gamma_{12}\right)\right|^{2}+\left|E_{p, z}\left(\beta+\gamma_{12}\right)\right|^{2}},
$$

where the integration again runs over $x_{1}, x_{2}, \omega_{1}$, and $\omega_{2}$ and where we have now introduced $\beta=\frac{1}{2}\left(x_{1}-x_{2}-\Delta x\right)$ as help variable. The sensitivity of $V_{\text {odd }}$ to a transverse displacement $\Delta x$ is mainly determined by the exponential factor in Eq. (2), again in combination with the limitations set by the finite integration range over the detection apertures and pump profile. The "odd" geometry thereby probes the two-photon coherence in the difference coordinate $\delta x_{s}-\delta x_{i}$, whereas the "even" geometry probed its coherence in the sum coordinate $\delta x_{s}+\delta x_{i}$. The above result again applies to all geometries with an odd number of mirrors in the combined signal and idler paths; Eqs. (9) and (10) remain basically the same, apart from some trivial minus signs and a possible redefinition of $\beta$.

\section{Temporal labeling}

In this section we will discuss the temporal labeling in a HOM interferometer with perfectly aligned beams $(\Delta x=0)$, but unbalanced arm lengths $(\Delta t \neq 0)$. The calculated $V_{\mathrm{HOM}}(\Delta t)$ is different for the two generic case, where the total number of mirrors is either even or odd. Whereas the even case exhibits only temporal labeling, the odd geometry also exhibits a combined temporal and spatial labeling, which can reduce $V_{\mathrm{HOM}}$ even further.

We will start by analyzing the even case for a symmetric pump $\left[E_{p, z}(x)=E_{p, z}(-x)\right]$. Substitution of $\Delta x=0$ in Eq. (8) and removal of the spatial integration (under the assumption that the shift $\gamma_{12}$ does not affect this integration in any serious way) yields

$$
V_{\text {even }}(\Delta t)=\frac{\operatorname{Re}\left(\int d \omega_{1} e^{i\left(2 \omega_{1}-\omega_{p}\right) \Delta t} T_{1}\left(\omega_{1}\right) T_{2}\left(\omega_{p}-\omega_{1}\right)\right)}{\int d \omega_{1} T_{1}\left(\omega_{1}\right) T_{2}\left(\omega_{p}-\omega_{1}\right)},
$$

where $T_{1}$ and $T_{2}$ are the intensity transmission spectra of filters located in front of the detectors 1 and 2, respectively. We thus obtain the well-known result that the HOM dip has the same shape, but is twice as narrow as the Fourier transform of the product $T_{1}\left(\omega_{1}\right) T_{2}\left(\omega_{p}-\omega_{1}\right)$ [11]. For identical filters with a sharp block-shaped transmission spectrum of width $\Delta \omega_{f}$ centered around $\frac{1}{2} \omega_{p}$, Eq. (11) yields

$$
V_{\text {even }}(\Delta t)=\frac{\sin \left(\Delta \omega_{f} \Delta t\right)}{\Delta \omega_{f} \Delta t}
$$

The full width at half-maximum (FWHM) of this visibility function is $1.21 \pi / \Delta \omega_{f}=1.21 \lambda^{2} /\left(2 c \Delta \lambda_{f}\right)$. If the transmission spectra of the filters are not properly centered, the product $T_{1} T_{2}$ will sharpen up and the temporal coherence of the detected two-photon field will increase.

If the combined number of mirrors in the signal and idler path is odd, we should substitute $\Delta x=0$ in Eq. (10) instead of Eq. (8). It is now in general not possible to separate the spatial and spectral integration, because the displacement $\gamma_{12} \propto \Delta \omega_{12}$ appears both in the argument of $E_{p, z}$ and in the exponential factor $\exp \left[-\left(i 2 k_{p} / L\right) \gamma_{12} \beta\right]$. Separation is only possible in two cases: if either the detection apertures are small enough to sufficiently limit the integration range over $\beta$, or if the displacement $\gamma_{12}$ is sufficiently small, we retain the result we had for the even case [Eq. (12)].

We will first discuss the physical origin of this combined labeling, before quantifying what we mean with "sufficiently small." In general, the visibility $V(\Delta t)$ decreases when the time difference between the photons arriving at detector 1 and 2 allows one (even only in principle) to distinguish which photon took the signal path and which one took the idler path. The important point to note is that this time difference is only equal to the set value $\Delta t=\Delta L / c$ for photon pairs that originate from the center of the pumped region. Photon pairs that originate from the outer parts of the pumped region can experience an additional temporal delay of typically $\Delta t_{\text {extra }}= \pm 2 \Theta w_{p} / c$ between their signal and idler photon, for a Gaussian pump beam of waist $w_{p}$. This delay alone does not reduce the visibility, as the contributions on either side of the pumped area can compensate each other, and actually do so for the even case. For the odd case, this extra term can lead to a degradation of the visibility, but only if the integration in Eq. (10) is large enough, i.e., if the apertures are opened wide enough in comparison to the pump divergence. The degradation will be small only if $\Delta \omega_{f} t_{\text {extra }} \ll \pi$. This criterium roughly translates into $\Delta \omega_{f} / \omega_{p} \ll \theta_{p} / \Theta, \theta_{p}$ being the far-field opening angle of the pump laser.

From an experimental perspective, the extra term in $V_{\text {odd }}$ makes two-photon interferometers with an odd number of mirrors more difficult to operate than interferometers with an even number of mirrors. In practice, great care must be taken to avoid the mentioned additional labeling. A two-photon interferometer with an odd number of mirrors will only provide a good visibility for apertures much larger than the pump size if three conditions are satisfied: (i) the spectral filters should be narrow enough, (ii) the opening angle $\Theta$ should be small enough, and (iii) the pumped region should be compact enough. Together these three conditions translate into the requirement that the dimensionless ratio of the detection bandwidth over the pump frequency should be much smaller than the ratio of the pump divergence over the open- 
ing angle, i.e., $\Delta \omega_{f} / \omega_{p} \ll \theta_{p} / \Theta$. If this is not the case, the combined spatial and spectral labeling will lead to a reduction of $V_{\text {odd }}(\Delta t=0)$ and a widening of the $V_{\text {odd }}(\Delta t)$ profile as compared to Eq. (12). The precise amount of which depends mainly on the dimensionless product $\left(\Delta \omega_{f} / \omega_{p}\right)\left(\Theta / \theta_{p}\right)$ and to a lesser extent on the position of the detectors in relation to the near or far field of the pump.

\section{E. Spatial labeling}

Next we will discuss spatial labeling in a HOM interferometer with balanced arms $(\Delta t=0)$ and sufficiently narrow spectral filters to validate the quasimonochromatic $(\Delta \omega=0)$ limit. We again distinguish between interferometers with an even and odd number of mirrors.
For the "even" case, Eq. (8) can be easily solved if the integration range over $x_{1}$ and $x_{2}$ is large enough to change it into an effective integration of $x_{1}+x_{2}$ and $x_{1}-x_{2}$ over $[-\infty, \infty]$. The integration simplifies even further when one realizes that the overlap $\langle\psi \mid \phi\rangle$ between two wave functions $|\psi\rangle$ and $|\phi\rangle$ does not change upon propagation, due to the unitary character of the propagator $h\left(x, x^{\prime}\right)$. The visibility $V_{\text {even }}(\Delta x)$ is thereby found to be a direct measure for the overlap of the pump profile with a displaced version thereof. If this pump profile is a fundamental Gaussian function with beam waist $w_{p}$, we obtain the simple result

$$
V_{\text {even }}(\Delta x)=\exp \left(-\frac{1}{2} \Delta x^{2} / w_{p}^{2}\right) \text {. }
$$

For the "odd" case, we must substitute $\Delta t=0$ and $\Delta \omega=0$ in Eq. (10) instead of Eq. (8) to obtain

$$
V_{\text {odd }}(\Delta x) \approx \frac{\operatorname{Re}\left[\left.\iint d x_{1} d x_{2}\left|E_{p, z}\left(\frac{1}{2}\left(x_{1}-x_{2}+\Delta x\right)\right)\right|\right|^{2} \exp \left(\frac{i k_{p}}{2 L}\left(x_{1}+x_{2}\right) \Delta x\right)\right]}{\iint d x_{1} d x_{2}\left|E_{p, z}\left(\frac{1}{2}\left(x_{1}-x_{2}+\Delta x\right)\right)\right|^{2}} .
$$

If the aperture diameters are much larger than the size of the pump beam in the detection plane, we can again rewrite the integrations over $x_{1}$ and $x_{2}$ into integrations over $x_{1}+x_{2}$ and $x_{1}-x_{2}$ and use $x_{1} \approx x_{2}$ as the outcome of the latter integration to obtain

$$
\begin{aligned}
V_{\text {odd }}(\Delta x) & \approx \frac{\operatorname{Re}\left[\int d x_{1} d y_{1} \exp \left(\frac{i k_{p}}{L} x_{1} \Delta x\right)\right]}{\int d x_{1} d y_{1}} \\
& \approx \frac{2 J_{1}\left(\pi d \Delta x /\left(\lambda_{p} L\right)\right)}{\pi d \Delta x /\left(\lambda_{p} L\right)} .
\end{aligned}
$$

In the final step, we have expressed the integration over a circular aperture with diameter $d$ in terms of the first-order Bessel function $J_{1}$. We define the typical transverse coherence length $\Delta x_{\mathrm{coh}}$ as the full width at half-maximum (FWHM) of $V_{\text {odd }}(\Delta x)$, which is 1.16 times the peak-to-zero width of $\Delta x=1.22 L\left(\lambda_{p} / d\right)$. The sensitivity of a two-photon interferometer with an odd number of mirrors to transverse displacement is thus found to be determined solely by the size of the detecting apertures. More specifically, $V_{\text {odd }}(\Delta x)$ has the same shape, but is just twice as narrow, as the diffraction limit at the crystal found for a uniform but focused illumination of one of the detecting apertures with the detected wavelength $2 \lambda_{p}$.

To arrive at Eq. (15) we had to assume that the aperture sizes were large as compared to the size of the pump beam. If only one of the two apertures satisfies this criterium, we can still conveniently replace the integrations over $x_{1}$ and $x_{2}$ by integrations over $x_{1}+x_{2}$ and $x_{1}-x_{2}$ and solve the latter. For this case of asymmetric aperture sizes, the resulting Eq. (15) thus remains valid. If the apertures have equal sizes, but are not very large as compared to the size of the pump beam, the aperture diameter in Eq. (15) should roughly be reduced from its physical size $d$ to an effective size $d_{\text {eff }} \approx d-w$ to account for the reduced detection efficiency of photon pairs that fall close to the edge of either aperture. Here, $w$ is the size of the pump beam in the detection plane and thereby one-half of the positional spread in one photon for a fixed position of the other photon.

\section{EXPERIMENTAL RESULTS}

\section{A. Experimental setup}

Our experimental setup, representing a two-photon (Hong-Ou-Mandel type) interferometer, is shown in Fig. 2. A $\mathrm{cw}$ krypton ion laser operates at a wavelength of $407 \mathrm{~nm}$ and emits $70 \mathrm{~mW}$ in a pure $\mathrm{TEM}_{00}$ mode. This light is mildly focused (measured opening angle typically $\theta_{p} \approx 0.50 \mathrm{mrad}$ and waist $w_{p} \approx 260 \mu \mathrm{m}$ ) on a 1 - $\mathrm{mm}$-thick type-I BBO crystal (cutting angle $29.2^{\circ}$ ). The crystal is tilted such that the emitted SPDC cone extends over a full opening angle of 2 $\times 1.6^{\circ}$ around the pump direction. Two entangled beams $s$ and $i$ (signal and idler), selected from this light cone by apertures behind a broadband beamsplitter at $1.20 \mathrm{~m}$ from the crystal, serve as input channels of the beamsplitter. In one of the two input beams, a reflecting open prism is placed on top of two perpendicularly mounted translation stages to enable accurate control of both the path-length difference $\Delta L$ and the transverse beam displacement $\Delta x$, using motorized 
actuators. In most of the experiments, the output beams of the beamsplitter are focused onto free-space single photon counters (Perkin Elmer SPCM-AQR-14) by $f=6 \mathrm{~cm}$ lenses located at $1.50 \mathrm{~m}$ from the crystal. We note that these counters still operate as good buckets under typical transverse beam displacements of $\Delta x=1 \mathrm{~mm}$ in our experiments as the demagnified displacement at the detector is then still only $\frac{6}{150} \Delta x=40 \mu \mathrm{m}$ whereas the active area of the detector is typically $200 \mu \mathrm{m}$ in diameter. Though omitted in Fig. 2 for simplicity, our scheme allows an easy switch between freespace and fiber-coupled counters (Perkin Elmer SPCM$\mathrm{AQR}-14-\mathrm{FC}$ ), connected to single-mode fibers (numerical aperture of 0.12) and 10× objectives. Bandwidth selection is done by interference filters (10 nm FWHM) in combination with red filters (Melles Griot RG715). An electronic circuit records coincidence counts within a time window of $1.76 \mathrm{~ns}$.

In order to achieve the precise temporal alignment that a HOM interferometer requires, i.e., simultaneous arrival of entangled pair-photons at the beamsplitter, we use a similar trick as presented in Ref. [20]. We employ a flip mirror to inject light from a diode laser (visible wavelength $\approx 640 \mathrm{~nm}$ ) into the setup, such that its emitted light virtually covers both signal and idler paths (see Fig. 2). By tuning this laser below threshold, where it acts as a bright LED with a limited coherence length, the path-length difference can be set to within a few $\mu \mathrm{m}$. Final finetuning of the path-length difference and the angular alignment between the two beams (within a few $\mu \mathrm{rad}$ ) is done by motorized actuators (Newport LTA-HL; submicron stepsizes) attached to both translation stages and beamsplitter.

In our main experiments, we measure the coincidence count rate as a function of the time delay $\Delta t=\Delta L / c$ and relative beam displacement $\Delta x$ between the signal and idler beam, in order to quantify the two-photon temporal and spatial coherence, respectively. We have employed both an even and an odd number of mirrors to demonstrate the essential role of the mirror number in two-photon HOM interference. Most of our measurements are however done with the odd configuration (see Fig. 2) as this is the most unexplored case. Furthermore, we have applied free-space detection behind both $4 \mathrm{~mm}$ and $14 \mathrm{~mm}$ apertures, corresponding to detection angles of $\theta_{\mathrm{det}}=1.7 \mathrm{mrad}$ and $\theta_{\mathrm{det}}=5.8 \mathrm{mrad}$, respectively. These values are well within the angular width of the SPDC ring of $\theta_{\mathrm{SPDC}}=18 \mathrm{mrad}$ that we calculate and observe for our (type-I) geometry. In addition, we use spectral filters with bandwidths that are much narrower than the generated SPDC bandwidth $(>50 \mathrm{~nm})$. These two conditions ensure operation in the thin-crystal limit.

\section{B. Temporal labeling}

In Fig. 3(a) the measured coincidence count rate behind $14 \mathrm{~mm}$ apertures is plotted versus time delay $\Delta t$. Fitting the data points with Eq. (12) yields a full width at half-maximum of $133 \pm 2$ fs. For $4 \mathrm{~mm}$ apertures we obtain the same value. These values agree very well with the theoretical coherence time of $133 \mathrm{fs}$, calculated for a block-shaped transmission filter with a measured spectral bandwidth of $\Delta \lambda=10 \mathrm{~nm}$ centered around $\lambda=814 \mathrm{~nm}$. The observed sidelobe structure is
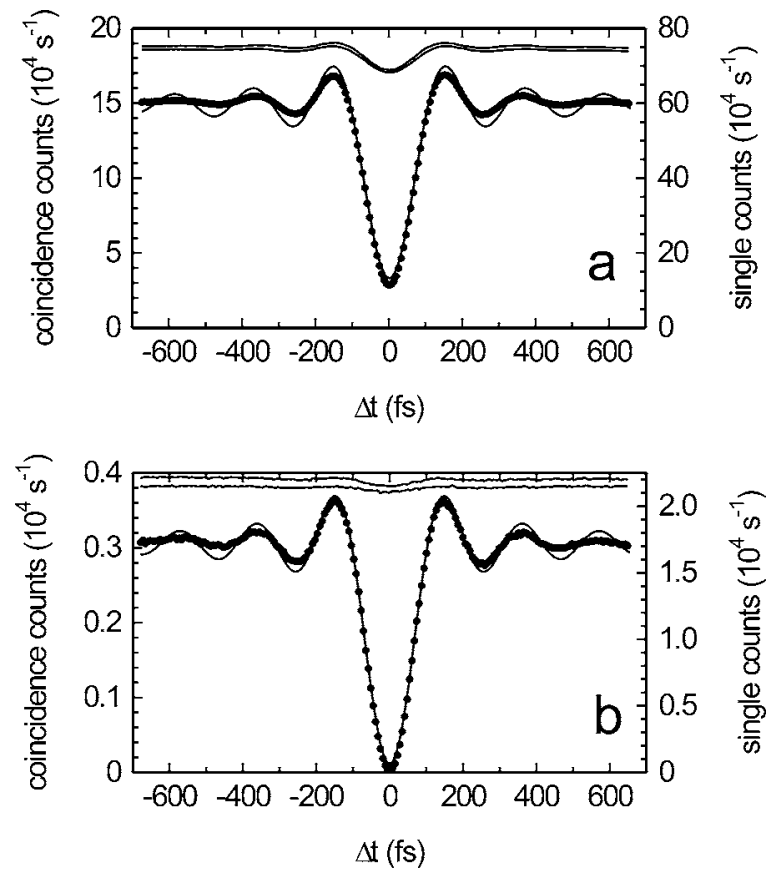

FIG. 3. Two-photon temporal coherence, measured as the coincidence count rate (dots) versus time delay $\Delta t$, for (a) free-space detection behind $14 \mathrm{~mm}$ apertures and (b) fiber-coupled detection. Sinc-shaped fits and the measured single count rates (solid curves; righthand scale) are plotted as well.

Fourier related to the spectral cutoff produced by the sharpedged interference filters. Slight deviations between data points and fits are attributed to the nonperfect block shape of the filter transmission function.

The quality of the two-photon interference can be quantified by the measured peak visibilities, being $V$ $=(85.0 \pm 0.5) \%$ and $V=(81.0 \pm 0.5) \%$ for $4 \mathrm{~mm}$ and $14 \mathrm{~mm}$ apertures, respectively. For fiber-coupled detection, we measure a much higher visibility of $V=(94.0 \pm 0.5) \%$. This value is very close to the theoretical limit of $V_{R T}=95 \%$ of our beamsplitter, having a measured $T / R$ ratio of 58/42. Figure 3(b) shows the temporal coherence measured with fibercoupled detectors scheme but now with a better high-quality 50-50 laserline beamsplitter. We again obtain a FWHM of $133 \pm 2 \mathrm{fs}$, but the peak visibility is considerably higher at $V=(99.3 \pm 0.2) \%$. The lower peak visibilities obtained with free-space detection is attributed to the spatial labeling observed by the bucket detectors (see Figs. 5 and 6).

Apart from the coincidence dips, Fig. 3 also shows prominent dips in the measured single count rates. The occurrence of a single dip has first been reported by Resch et al. [21]. This extra dip occurs as a result of the limitation of a photodetector to record two simultaneously arriving pair-photons as two single clicks. As these arrivals are more numerous for a balanced HOM interferometer than for an unbalanced one, a dip will show up in the measured single count rate as well.

In Fig. 4 we highlight the single dip that we measured behind $14 \mathrm{~mm}$ apertures [data copied from Fig. 3(a)]. This data is of much higher quality than the one presented in Ref. [21]; though sampling only $10 \mathrm{~s}$ for each data point, we obtain a statistical error of $<0.1 \%$ that is even too small to 


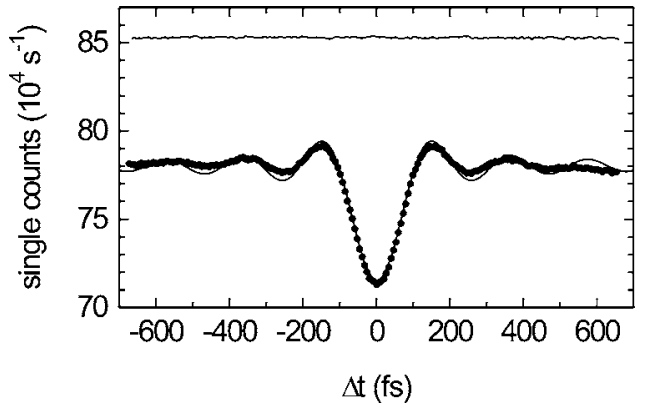

FIG. 4. Single count rate measured in a HOM experiment (dots) with sinc-shaped fit (detail of Fig. 3(a)). The solid curve shows the sum of the single count rates measured when either the signal or the idler path is blocked. All displayed count rates are corrected for $50 \mathrm{~ns}$ deadtime of the detector.

display. This allows us to observe the clear sinc-shaped profile identical to the coincidence dip with a FWHM of $133 \pm 2$ fs. Based on measured rates of $7.13 \times 10^{5} \mathrm{~s}^{-1}$ and $7.81 \times 10^{5} \mathrm{~s}^{-1}$ at zero and infinite delay, respectively, we determine a dip visibility of $V_{\mathrm{sc}} \approx 9 \%$. A calculation from $V_{\mathrm{sc}}$ $=V \eta /(4-\eta)[21]$ yields the same value, thereby using $V$ $=81 \%$ and an overall detection efficiency of $\eta=0.40$, as deduced from the measured quantum efficiency (coincidence to single ratio) of $\eta_{q}=0.20$. All count rates shown in Fig. 4 have been multiplied by a factor of $1 /\left(1-\tau_{d} R_{\text {det }}\right) \approx 1.04$ to correct for the detector deadtime of $\tau_{d}=50 \mathrm{~ns}$ and compare with the calculation mentioned above.

To further illustrate the origin of the single dip, we have also plotted the sum of the measured single count rates in absence of HOM interference as the solid curve in Fig. 4. This rate of $8.54 \times 10^{5} \mathrm{~s}^{-1}$ shows no dip as it is obtained by adding the individual signal and idler rates of 5.00 $\times 10^{5} \mathrm{~s}^{-1}$ and $3.54 \times 10^{5} \mathrm{~s}^{-1}$, where the rate imbalance is due to the beamsplitter ratio $T / R=58 / 42$. We thus measure a single count rate reduction of $16.5 \%$ for the balanced interferometer $(\Delta t=0)$, but also obtain an $8.5 \%$ reduction in $a b$ sence of HOM interference $(\Delta t=\infty)$. This latter reduction of course results from a random $1 / 4$ probability that both photons arrive at the detector under study. At a finite detection efficiency $\eta$ we expect the single count rate to be reduced by a factor $(1-\eta / 4)$ and $[1-\eta / 4(1+V)]$ in an interferometer off and on resonance, as compared to the sum of the individual rates. For our conditions of $V=81 \%$ and $\eta=0.40$, we expect reductions of $(1+V) \eta / 4=18 \%$ and $\eta / 4=10 \%$ for the balanced and unbalanced interferometer, respectively, which agree reasonably well with the measured values.

As an aside we note that our count rates are large enough to experience some visibility reduction through the influence of double photon pairs. We estimate this reduction to be $\Delta V=8 R_{c} \tau_{c c}\left(1 / \eta^{2}-1 / 2 \eta\right)$, based on a generated pair rate $R$ $=2 R_{c} / \eta^{2}$ and a coincidence time window $\tau_{c c}$. Our measured visibility of $V=78 \%$ for $17 \mathrm{~mm}$ apertures is expected to suffer from a reduction of only $\Delta V \approx 1 \%$, based on a measured coincidence rate of $R_{c}=2.0 \times 10^{5} \mathrm{~s}^{-1}$ and $\eta=0.40$. To check that higher coincidence rates lead to larger reductions, we have also used a $4 \mathrm{~mm}$ crystal. At a measured rate of $R_{c}$ $=8 \times 10^{5} \mathrm{~s}^{-1}$ we measure a lower visibility of $V=73 \%$,

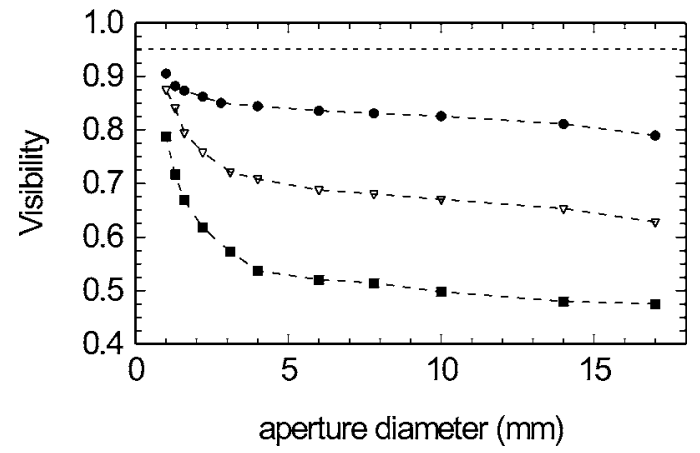

FIG. 5. Measured peak visibility $V_{\text {odd }}$ versus aperture diameter (at $1.2 \mathrm{~m}$ from crystal) for $\Delta \lambda=10 \mathrm{~nm}$ interference filters and three different pump sizes: $\mathrm{w}_{p}=260 \mu \mathrm{m}$ (dots), $\mathrm{w}_{p}=400 \mu \mathrm{m}$ (triangles) and $\mathrm{w}_{p}=700 \mu \mathrm{m}$ (squares). The dashed horizontal line at $V=95 \%$ indicates the visibility limit set by the beamsplitter $T / R$ ratio of $58 / 42$.

which is indeed compatible with the expected reduction of $\Delta V \approx 5 \%$.

The theory in Sec. II D predicts that the peak visibility in a HOM interferometer with an odd number of mirrors can be limited by a combined temporal and spatial labeling that depends on three different parameters: the aperture size, the pump size at the crystal and the detected spectral bandwidth. The first two limitations are demonstrated in Fig. 5, which shows the measured visibility as a function of the aperture diameter for three pump sizes $w_{p}$, using a $\Delta \lambda=10 \mathrm{~nm}$ interference filter. The largest pump spots yield the lowest visibilities, as expected. Note how the visibilities increase steeply for the smallest apertures where diffraction removes the spatial labeling.

An increase of the pump spot not only leads to a reduction of the peak visibility but also to a widening of the $V_{\mathrm{HOM}}(\Delta t)$ curve. At an aperture size of $14 \mathrm{~mm}$ we measure (FWHM) coherence times of 133 fs for $w_{p}=260 \mu \mathrm{m}, 147$ fs for $w_{p}$ $=400 \mu \mathrm{m}$, and $180 \mathrm{fs}$ for $w_{p}=700 \mu \mathrm{m}$, all at $\Delta \lambda=10 \mathrm{~nm}$. For these three geometries the dimensionless quantity $\left(\Delta \omega_{f} / \omega_{p}\right)\left(\Theta / \theta_{p}\right)$ that quantifies the extra labeling increases from 0.34 to 0.49 and 0.86 .

The limitation of the visibility by the detected spectral bandwidth is shown in Fig. 6, where the measured visibility is plotted versus aperture size for both $\Delta \lambda=5 \mathrm{~nm}$ and $10 \mathrm{~nm}$ interference filters, and a pump waist of $w_{p}=260 \mu \mathrm{m}$. The narrower filters yield higher visibilities. All observations made in relation to Figs. 5 and 6 are compatible with the prediction made in Sec. II D on combined temporal and spatial labeling. For an even number of mirrors in our interferometer (with one extra mirror in signal path; see below) we have observed none of these combined-labeling effects, again in agreement with Sec. II D.

\section{Spatial labeling}

As our key experiment we have measured the spatial coherence of the generated two-photon wave packet. Figures 7(a) and 7(b) show the coincidence count rate measured as a function of the relative transverse beam displacement $\Delta x$ for 


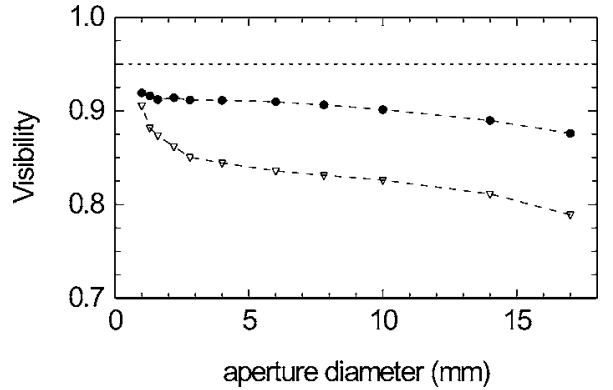

FIG. 6. Measured peak visibility $V_{\text {odd }}$ versus aperture diameter for $\Delta \lambda=5 \mathrm{~nm}$ (solid dots) and $\Delta \lambda=10 \mathrm{~nm}$ interference filters (triangles), and a pump size of $\mathrm{w}_{p}=260 \mu \mathrm{m}$. The dashed horizontal line at $V=95 \%$ indicates the visibility limit set by the beamsplitter $T / R$ ratio of 58/42. The error margins of 0.005 in the vertical scale are too small to display.

$4 \mathrm{~mm}$ and $14 \mathrm{~mm}$ apertures, and perfect temporal coherence $(\Delta t=0)$. Fitting the data points with Eq. (4) yields (FWHM) transverse coherence lengths of $\Delta x_{\text {coh }}=184 \pm 10 \mu \mathrm{m}$ and $\Delta x_{\text {coh }}=54 \pm 4 \mu \mathrm{m}$, respectively. These values are only slightly larger than the values of $\Delta x_{\mathrm{coh}}=175 \mu \mathrm{m}$ and $50 \mu \mathrm{m}$, expected from Eq. (15). We ascribe these minor deviations to a reduced detection efficiency of photon pairs close to the aperture edges, which leads to effectively smaller aperture sizes and thus increased coherence lengths. This correction disappears if we employ the asymmetric geometry of a $4 \mathrm{~mm}$ aperture in one arm and a $14 \mathrm{~mm}$ one in the other, and perform the same measurement [see Fig. 7(c)]. We then indeed obtain a somewhat smaller transverse coherence length of $166 \pm 10 \mu \mathrm{m}$ that is solely determined by the smallest aperture. Our measurements clearly demonstrate that two-photon interference measured behind smaller apertures results in a larger spatial coherence length, and vice versa.

The observations that a transverse displacement in one of the beams leads to a reduction of the two-photon interference can be easily understood in terms of spatial labeling. This is schematically shown in Fig. 1, where the upper circle depicts the pumped area at the crystal. The four lower circles depict images of this pumped area that can potentially be made at both detectors if the appropriate lenses are used (for simplicity we assume perfect imaging without inversion). These images are represented by solid and dashed circles corresponding to whether the photons have traveled the signal (solid) or idler (dashed) path, respectively. Consequently, a solid circle at detector 1 matches a dashed circle in detector 2 , and vice versa. The transverse displacement $\Delta x$ of the idler beam is shown as light-dashed lines.

Now suppose we detect a photon at detector 1 at the lower-left cross-mark. Tracing this photon back results in two different birth positions (cross-marks in upper circle) separated by $\Delta x$ at the crystal plane. Tracing its partner photon back to detector 2 then yields two possible imaging positions (lower-right cross-marks) in circle $s_{2}$ and $i_{2}$, separated by $2 \Delta x$. If the resolution of our imaging system is good enough to distinguish between these two possibilities, the "which-path" information provided by this spatial labeling will destroy the two-photon interference. As diffraction by the apertures limits the distinguishability, larger transverse
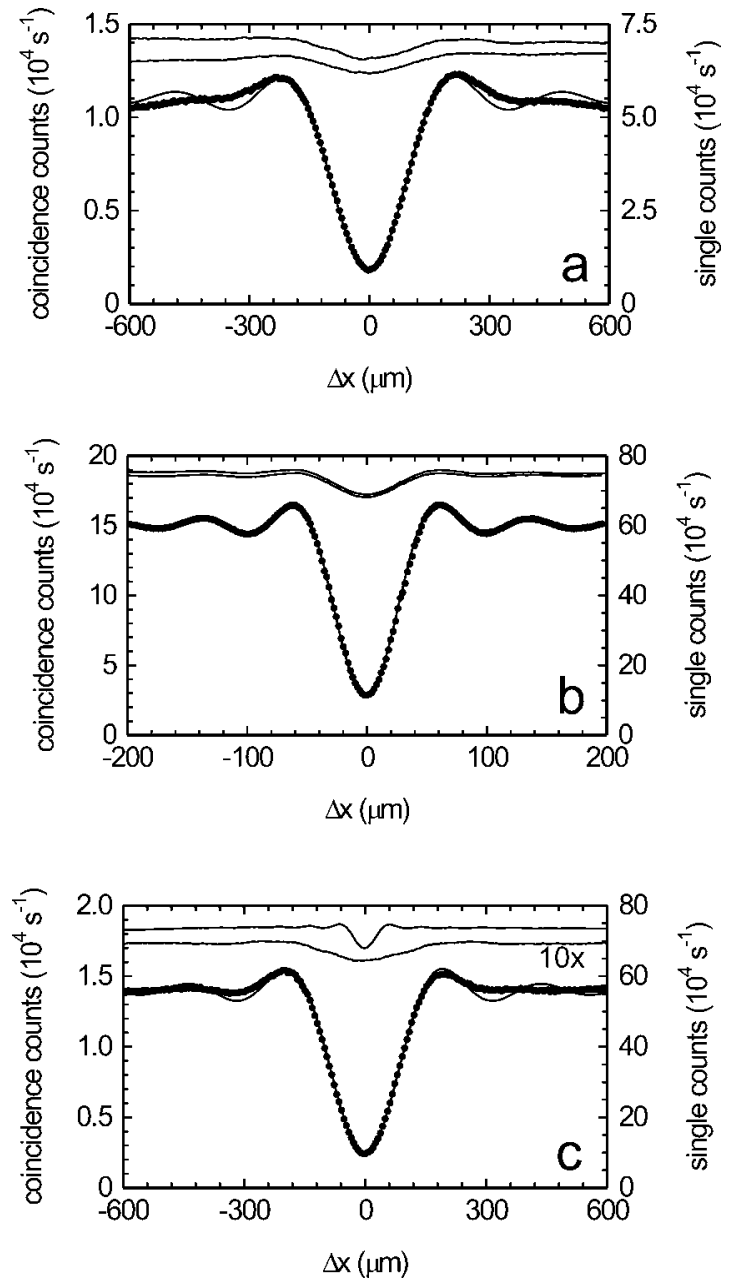

FIG. 7. Two-photon spatial coherence, measured as the coincidence count rate (dots) versus relative transverse displacement behind (a) $2 \times 4 \mathrm{~mm}$, (b) $2 \times 14 \mathrm{~mm}$ and (c) $4+14 \mathrm{~mm}$ apertures. The solid curves represent the measured single count rates and fits of the coincidence count rates. Especially, the fit in (b) is of excellent quality. The lower single count rate in (c), which was measured behind the $4 \mathrm{~mm}$ aperture, has been multiplied by a factor of 10 in order to visualize the dip-structure. Note the differences in the horizontal scales.

coherence lengths will be attained with smaller apertures, and vice versa. As we need the combined positional information of both photons to decide upon their paths, the diffraction limit of the smallest of the two apertures will largely determine the observed coherence length. As an aside, we note that a similar reasoning can be applied to the results in Ref. [15], where large apertures correspond to a small diffraction limit, good distinguishability between the two probability paths, and a low HOM visibility.

We will next focus our attention on Fig. 7(c), which refers to an asymmetric interferometer with apertures of $4 \mathrm{~mm}$ and $14 \mathrm{~mm}$ in front of the two detectors. At first thought, one might expect the single dip to follow the coincidence dip, irrespective of the aperture geometry. This is however not the case: we measure different widths (FWHM) of 190 $10 \mu \mathrm{m}$ and $54 \pm 4 \mu \mathrm{m}$ for the single dips behind the $4 \mathrm{~mm}$ and $14 \mathrm{~mm}$ aperture, respectively, whereas the coincidence width 

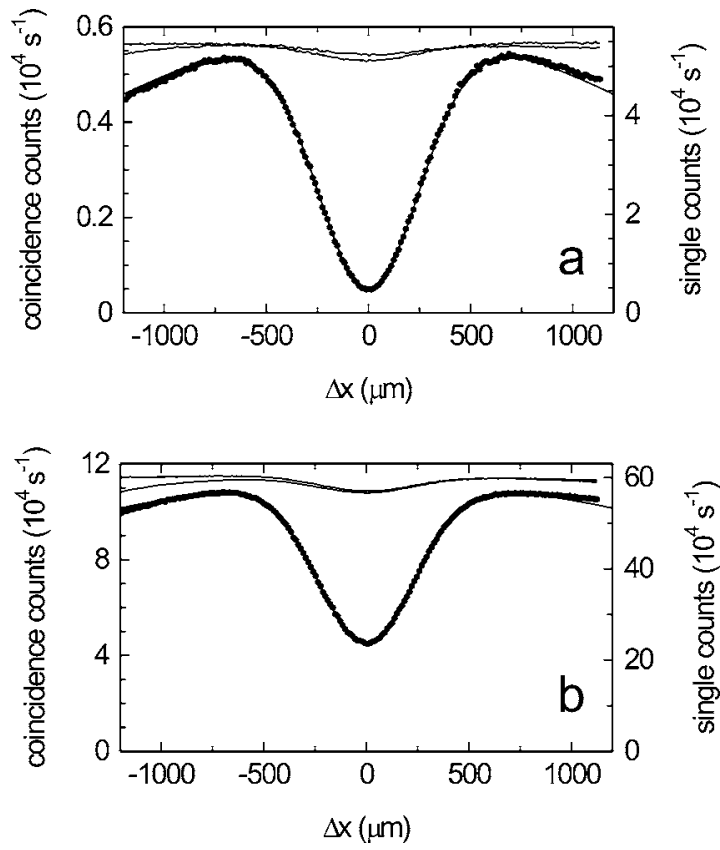

FIG. 8. Two-photon spatial coherence for an even number of mirrors. The coincidence count rate (dots) is plotted versus relative transverse displacement behind (a) $4 \mathrm{~mm}$ and (b) $14 \mathrm{~mm}$ apertures. Coincidence counts fits and single count rates (solid curves) are plotted as well.

is $166 \pm 10 \mu \mathrm{m}$. These values are practically the same as the widths of the single and coincidence dips observed for a symmetric setup with $2 \times 4 \mathrm{~mm}$ and $2 \times 14 \mathrm{~mm}$ apertures, respectively [see Figs. 7(a) and 7(b)].

The intriguing asymmetry in the single dips can be understood as follows. Pair-photons originating from those parts of the signal and idler beam that are captured by the $14 \mathrm{~mm}$ aperture but not by the $4 \mathrm{~mm}$ one, will be registered only by the detector behind the larger aperture. Simultaneous arrivals of these photons due to bunching will therefore affect only the single dip measured with this detector, but will not contribute to the coincidence dip. As photon bunching occurs within a smaller range of transverse displacements for larger apertures, the measured single dip for the $14 \mathrm{~mm}$ aperture in Fig. 7(c) is as narrow as the coincidence dip that would be measured with $14 \mathrm{~mm}$ apertures in both output channels. Consequently, the $4 \mathrm{~mm}$ aperture single dip in the same figure is almost as broad as the measured coincidence dip.

To demonstrate that the two-photon spatial coherence is very different for interferometers with an even or odd number of mirrors, we have added a second mirror in the signal path, using now six $(2+4)$ mirrors in total. In Figs. 8(a) and 8 (b) we have plotted the coincidence rate versus the transverse displacement $\Delta x$, measured in this even geometry for $2 \times 4 \mathrm{~mm}$ and $2 \times 14 \mathrm{~mm}$ apertures, respectively. The coincidence dips are fit with the profile $a \exp \left[-(\Delta x)^{2} / b^{2}\right][1$ $\left.-c \exp (\Delta x)^{2} / 2 v^{2}\right]$, where the fit parameter $v$ is expected to yield the same near-field waist $w_{p}$ of the Gaussian pump profile for both aperture sizes. We indeed obtain similar widths of $v=253 \mu \mathrm{m}$ and $v=237 \mu \mathrm{m}$ for $4 \mathrm{~mm}$ and $14 \mathrm{~mm}$ apertures, respectively. These values agree well with the measured pump waist of $w_{p} \approx 260 \mu \mathrm{m}$. The exponential pre-

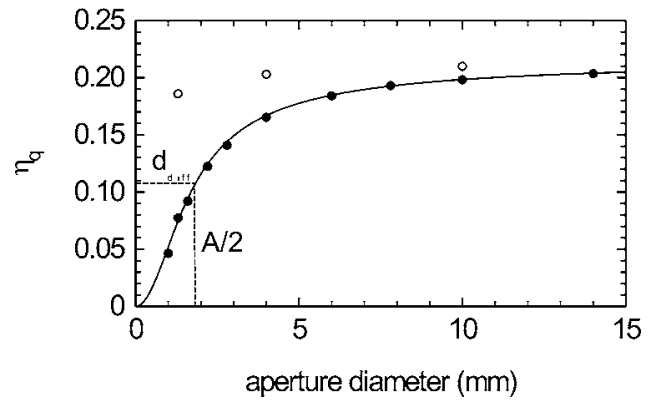

FIG. 9. Measured quantum efficiency $\eta_{\mathrm{q}}$ versus aperture diameter for equal apertures (solid dots), and for a geometry with one aperture fully open (open circles). The fit (solid curve) yields an asymptotic value of $A=0.217$ and a pump beam waist at the aperture plane of $\mathrm{w}=0.63 \mathrm{~mm}$.

factor roughly quantifies how the observed coincidence rates decreases when very large beam displacements shift the light outside the active area of the detectors. For this even geometry, we have measured $20 \%$ lower single count rates as compared to the odd geometry [see Figs. 7(a) and 7(b)] because of the increased crystal-aperture distance from $1.20 \mathrm{~m}$ to $1.37 \mathrm{~m}$.

In contrast to the odd geometry, the above result clearly shows that the two-photon spatial coherence for an even number of mirrors is only determined by the pump beam profile and is insensitive to the aperture size. The picture of spatial labeling, shown in Fig. 1 for the odd geometry, can also be applied to the even geometry. If we observe a certain photon position at detector 1 (lower-left cross-mark), we can again reconstruct two similar birth positions of this photon at the crystal (upper cross-marks). However, we now find only one position for the corresponding photon at detector 2 , as the $s_{2}$ and $i_{2}$ positions lie precisely on top of each other. This means that, irrespective of the aperture size, one cannot distinguish which probability channel (double reflection or double transmission) the pair-photons has traveled by judging from the detected positions of the partner photon. As the spatial labeling is only contained in the different birth positions for this even geometry, the which-path information comes now from the pump beam profile and is no longer determined by the aperture size if the later is much larger than $w$. Only the spatial symmetry of the pump beam and a possible transverse displacement $\Delta x$ matter.

\section{Modal analysis of spatial entanglement}

Next we will analyze the two-photon field in terms of a finite number of discrete modes. The shape of the pump laser defines a natural basis for this discrete modal analysis. This natural size will show up in an experiment where one fixes the position of one photon and measures the positional spread $\theta_{\text {diff }}=2 \theta_{p}$ of its partner photon in coincidence imaging $[18,19]$. To determine this natural size, we have performed a different experiment instead, where we vary the size of both apertures, working in a symmetric situation at (much) higher count rates. The solid dots in Fig. 9 depict the measured quantum efficiency $\eta_{q}$, being defined as the ratio of the coincidence count rate over the single rate, as a function of the 
aperture diameter $d$. The sharp decrease in $\eta_{\text {aq }}$ at small apertures results from the positional spread within the photon pair that was mentioned above. This spread is solely determined by the shape of the pump profile and can be fit with the expression [22]

$$
\eta_{q}(d)=\frac{A}{1+2 w^{2} / d^{2}}\left(1-\frac{\sqrt{\pi} \operatorname{erf}\left[\sqrt{1+d^{2} /\left(2 w^{2}\right)}\right]}{2 \sqrt{1+d^{2} /\left(2 w^{2}\right)}}\right),
$$

where the asymptotic value $A$ and the pump beam waist $w$ at the aperture plane $(1.2 \mathrm{~m}$ from crystal in our case) are fitting parameters. The diameter of $d_{\text {diff }}=1.8 \mathrm{~mm}$ where the measured quantum efficiency is $50 \%$ of its asymptotic value (see Fig. 9) gives the typical size of the fundamental transverse mode. The solid curve is a fit based on $A=0.217$ and $w$ $=0.63 \mathrm{~mm}$. The latter value agrees well with a calculated waist at the aperture plane of $w=0.65 \mathrm{~mm}$, that is based on a Rayleigh range of $z_{R}=0.52 \mathrm{~m}$, a near-field pump waist of $w_{p}=260 \mu \mathrm{m}$, and a pump opening angle of $\theta_{p}=0.50 \mathrm{mrad}$; these numbers are obtained from a measured pump waist of $w_{z}=1.8 \mathrm{~mm}$ at $z=3.6 \mathrm{~m}$ from the crystal. The SPDC diffraction angle $\theta_{\text {diff }}=2 \theta_{p}$ (SPDC wavelength $\lambda=2 \lambda_{p}$ ) will be used below for the calculation of the mode number.

The number of transverse modes detectable behind a farfield aperture of radius $a$ and angular size $\theta_{\mathrm{det}}=a / L$ is $N$ $=N_{1 \mathrm{D}}^{2}$, where the one-dimensional mode number

$$
N_{1 \mathrm{D}} \approx \frac{\theta_{\mathrm{det}}}{\theta_{\mathrm{diff}}}=\pi \frac{a_{p} a}{\lambda L},
$$

$a_{p}$ being the radius of the pump spot at the crystal, i.e., the near-field radius of the SPDC radiation. The approximation sign is related to the precise definition of the mode size (FWHM, Gaussian or sharp edge).

The second equality of Eq. (17) enables an easy link to a different measure for the number of interfering transverse modes, being the well-known Fresnel number $N_{F}$ given by

$$
N_{F}=\frac{a^{2}}{\lambda L} \approx \frac{a}{2.8 \Delta x_{\mathrm{coh}}} .
$$

Here $\Delta x_{\text {coh }}$ is the (FWHM) transverse coherence length that we defined below Eq. (15), and the prefactor $1 / 2.8$ $\approx 1.16 \times 1.22 / 4$ results from our definition of $\Delta x_{\text {coh. }}$. For a one-photon field the Fresnel number denotes the number of Fresnel zones that contribute, with alternating signs, to the field transmitted through a rotational symmetric aperture. A comparison between the two quantities defined in Eq. (17) and Eq. (18) yields $N_{F}=N\left(L / z_{R}\right)(2 / \pi)$, where $z_{R}=\frac{1}{2} k_{p} w_{p}^{2}$ is the Rayleigh range of the pump. As we typically work at $L / z_{R} \approx 2.3$, the numbers $N$ and $N_{F}$ should be comparable.

From our experimental results we can estimate the mode number $N$ and Fresnel number $N_{F}$ in three different ways. First of all, we can use Eq. (17) and divide the detection angle $\theta_{\text {det }}$ by the measured diffraction angle $\theta_{\text {diff }}$ to find $N$ $\approx 3$ and $N \approx 34$ for $4 \mathrm{~mm}$ and $14 \mathrm{~mm}$ apertures, respectively. Second, we can use Eq. (18) and compare the measured transverse coherence length $\Delta x_{\text {coh }}$ to the aperture size to obtain Fresnel numbers $N_{F} \approx 4$ and $N_{F} \approx 46$ for $4 \mathrm{~mm}$ and $14 \mathrm{~mm}$ apertures, respectively. The third measure for the transverse mode number can be deduced by comparing the single count rates shown in Figs. 3(a) and 3(b). As fibercoupled detection per definition addresses a single transverse mode, division of these mentioned count rates yields a mode number of $N=34$. A similar exercise for a $4 \mathrm{~mm}$ aperture (not shown) yields $N=7 \times 10^{4} / 2.1 \times 10^{4} \approx 3$. These numbers compare well with the mode numbers $N$ from the first estimate. All estimates show that our experiment addresses typically 4 or 40 modes for the 4 or $14 \mathrm{~mm}$ apertures, respectively.

\section{CONCLUDING DISCUSSION}

We have investigated the two-photon spatial coherence of entangled photon pairs by measuring the coincidence rate in a Hong-Ou-Mandel interferometer as a function of the relative transverse beam displacement for different aperture sizes. The calculated and observed coherence is completely different for an interferometer with an odd or even number of mirrors. For the odd case we have demonstrated that the transverse coherence length is inversely proportional to the aperture size. We also observed a well-defined dip in the single count rate and demonstrated the existence of a combined temporal and spatial labeling that can lead to a reduction of the HOM visibility under certain conditions. For the even case, we have shown that the transverse coherence length is basically determined by the pump waist.

\section{ACKNOWLEDGMENTS}

This work has been supported by the Stichting voor Fundamenteel Onderzoek der Materie. The authors thank J.P. Woerdman for stimulating discussions and Y.C. Oei for his assistance in the laboratory.
[1] A. Aspect, P. Grangier, and G. Roger, Phys. Rev. Lett. 47, 460 (1981).

[2] C. K. Hong, Z. Y. Ou, and L. Mandel, Phys. Rev. Lett. 59, 2044 (1987).

[3] P. G. Kwiat, K. Mattle, H. Weinfurter, A. Zeilinger, A. V. Sergienko, and Y. Shih, Phys. Rev. Lett. 75, 4337 (1995).

[4] N. Gisin, G. Ribordy, W. Tittel, and H. Zbinden, Rev. Mod. Phys. 74, 145 (2002).
[5] A. V. Sergienko and G. S. Jaeger, Contemp. Phys. 44, 341 (2003).

[6] T. B. Pittman, D. V. Strekalov, A. Migdall, M. H. Rubin, A. V. Sergienko, and Y. H. Shih, Phys. Rev. Lett. 77, 1917 (1996).

[7] J. G. Rarity and P. R. Tapster, Philos. Trans. R. Soc. London, Ser. A 355, 2267 (1997).

[8] T. Yamamoto, M. Koashi, S. K. Özdemir, and N. Imoto, Nature (London) 42, 343 (2003). 
[9] T. B. Pittman and J. D. Franson, Phys. Rev. Lett. 90, 240401 (2003).

[10] P. G. Kwiat, A. M. Steinberg, and R. Y. Chiao, Phys. Rev. A 45, 7729 (1992).

[11] Y.-H. Kim, J. Opt. Soc. Am. B 20, 1959 (2003).

[12] S. P. Walborn, A. N. de Oliveira, S. Pádua, and C. H. Monken, Phys. Rev. Lett. 90, 143601 (2003).

[13] D. P. Caetano and P. H. Souto Ribeiro, Phys. Rev. A 68, 043806 (2003).

[14] W. A. T. Nogueira, S. P. Walborn, S. Pádua, and C. H. Monken, Phys. Rev. Lett. 92, 043602 (2004).

[15] M. Atatüre, G. Di Giuseppe, M. D. Shaw, A. V. Sergienko, B. E. A. Saleh, and M. C. Teich, Phys. Rev. A 66, 023822 (2002).
[16] K. J. Resch, Phys. Rev. A 70, 051803(R) (2004).

[17] B. E. A. Saleh, A. F. Abouraddy, A. V. Sergienko, and M. C. Teich, Phys. Rev. A 62, 043816 (2000).

[18] C. H. Monken, P. H. Souto Ribeiro, and S. Padua, Phys. Rev. A 57, 3123 (1998).

[19] T. B. Pittman, D. V. Strekalov, D. N. Klyshko, M. H. Rubin, A. V. Sergienko, and Y. H. Shih, Phys. Rev. A 53, 2084 (1996).

[20] S. E. Spence, D. C. Stoudt, N. L. Swanson, A. D. Parks, and F. E. Peterkin, Rev. Sci. Instrum. 71, 23 (2000).

[21] K. J. Resch, J. S. Lundeen, and A. M. Steinberg, Phys. Rev. A 63, 020102(R) (2001).

[22] C. H. Monken, P. H. Souto Ribeiro, and S. Pádua, Phys. Rev. A 57, R2267 (1998). 